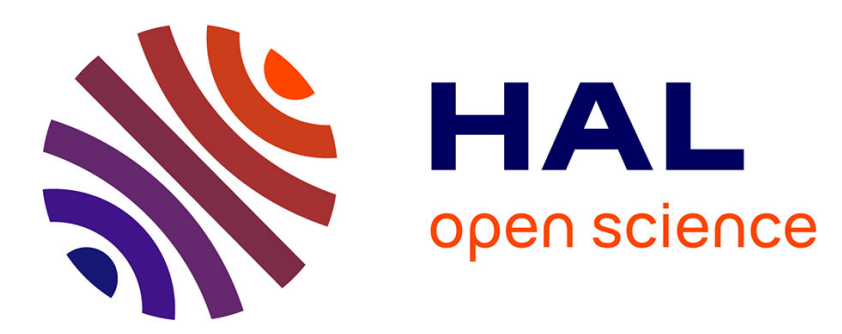

\title{
Appareillage de caractérisation de matériaux magnétiques pour l'électrotechnigue
}

\author{
J.-J. Rousseau, J.-P. Masson
}

\section{To cite this version:}

J.-J. Rousseau, J.-P. Masson. Appareillage de caractérisation de matériaux magnétiques pour l'électrotechnigue. Revue de Physique Appliquée, 1988, 23 (5), pp.947-954. 10.1051/rphysap:01988002305094700 . jpa-00245897

\section{HAL Id: jpa-00245897 https://hal.science/jpa-00245897}

Submitted on 1 Jan 1988

HAL is a multi-disciplinary open access archive for the deposit and dissemination of scientific research documents, whether they are published or not. The documents may come from teaching and research institutions in France or abroad, or from public or private research centers.
L'archive ouverte pluridisciplinaire HAL, est destinée au dépôt et à la diffusion de documents scientifiques de niveau recherche, publiés ou non, émanant des établissements d'enseignement et de recherche français ou étrangers, des laboratoires publics ou privés. 


\title{
Appareillage de caractérisation de matériaux magnétiques pour l'électrotechnique
}

\author{
J.-J. Rousseau et J.-P. Masson \\ Laboratoire de Composants de Puissance et Applications, Institut National des Sciences Appliquées, \\ 69621 Villeurbanne Cedex, France
}

(Reçu le 23 juillet 1987, révisé le 4 janvier 1988, accepté le 12 janvier 1988)

\begin{abstract}
Résumé. - Les constructeurs de machines électriques ont besoin lors de l'étude, puis de la fabrication, de relever les caractéristiques magnétiques précises des matériaux utilisés, quel que soit leur état de saturation. Cet article décrit un appareillage de mesure programmable permettant, non seulement d'accroître la précision, mais également de simplifier la mise en œuvre, rendant ainsi envisageable un contrôle en fabrication.
\end{abstract}

\begin{abstract}
The electrical machine manufacturers need for the design and the quality tests, to know with accuracy the magnetic characteristics relevant to the working conditions. This paper describes a measurement device for these characteristics. The use of a microprocessor allows the realization of a fast, accurate, and easy to use apparatus, so that the measurement can be done in industrial conditions.
\end{abstract}

\section{Introduction.}

L'industrie électrotechnique dispose actuellement de logiciels de calcul de champ magnétique utilisables dans le cas de matériaux saturables [1]. De plus les circuits magnétiques des machines de puissance fractionnaire travaillent à des inductions très élevées. Il est donc nécessaire de déterminer avec précision les caractéristiques magnétiques des circuits utilisés. Les traitements successifs subis par les tôles modifient les propriétés principalement de conduction électrique. Le contrôle des lots de tôles assemblées est donc également une opération souhaitable.

Nous avons choisi de réaliser un appareillage simple d'emploi permettant la mesure de cycles d'hystérésis quasi-statiques et la comparaison des courants induits d'un lot de fabrication à un autre. L'utilisation d'un micro-calculateur associé à un logiciel à menus successifs non seulement rend la conduite de l'essai très souple, avec l'évolution possible vers un automatisme presque total, mais permet une augmentation de la précision. Enfin, les mesures étant mémorisées, un programme de régression donne directement sous forme polynomiale, l'équation de la courbe de première aimantation.

Après avoir présenté le principe de mesure retenu et l'avoir comparé aux solutions classiquement adoptées, nous décrivons les particularités de construction de l'appareil et présentons des mesures obtenues sur des matériaux de conductibilités et perméabilités différentes.

\section{Principe de mesure.}

2.1 GÉNÉRALITÉS. - L'échantillon à tester est mis sous la forme d'un circuit magnétique fermé de section constante $S$. Un enroulement parcouru par un courant variable $I$ excite ce circuit.

La présence d'entrefer étant le plus possible à éviter, l'utilisation de sondes à effet Hall ou de magnéto-résistances n'est pas adaptée [2].

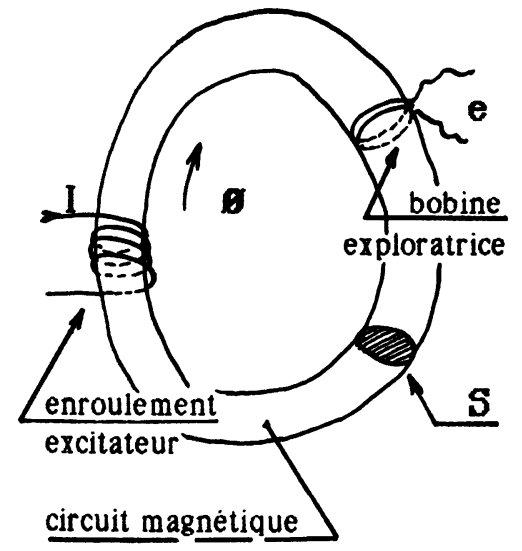

Fig. 1. - Principe de la mesure.

[Principle of measurement.] 
Un deuxième enroulement suivant au plus près le circuit magnétique est le siège d'une force électromotrice $e$ proportionnelle à la dérivée du flux le traversant.

Classiquement les hypothèses admises supposent qu'à un instant donné, dans toute section $S$ le flux $\phi$ est constant, uniforme, et égal à celui qui est embrassé par la bobine exploratrice [3]. Nous supposóns ces conditions remplies.

Outre l'utilisation de perméamètres, les circuits les plus utilisés sont les cadres EPSTEIN normalisés [4] et les tores.

Désirant nous placer dans les conditions les plus proches du produit fini (stator de machine asynchrone dans notre cas), nous utilisons l'empilage de tôles terminé, avec ses soudures d'assemblage, et dans un premier temps, supprimons les dents (Fig. 2). Nous disposons ainsi d'un cylindre de faible épaisseur par rapport à son diamètre qui représente un circuit à faibles fuites.

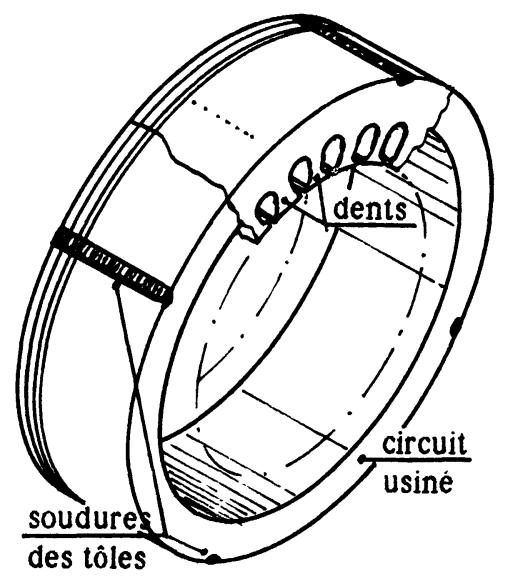

Fig. 2. - Elaboration du circuit de test en tôles au silicium.

[Implementation of the laminated circuit in test.]

2.2 ChOIX DE LA DYNAMIQUE DE MESURE. - Lors d'une variation du champ appliqué à un échantillon, interviennent simultanément le phénomène d'hystérésis et pour un matériau conducteur la circulation de courants induits. Ces courants se développent à l'échelle des domaines et à l'échelle de l'échantillon :

- pour des variations de flux très lentes, l'influence des courants induits est diminuée et on peut considérer que le cycle obtenu représente bien les propriétés statiques du matériau ; en particulier cette caractéristique sera utilisée pour déterminer les différentes perméabilités ;

- pour des variations rapides et connues du courant, la vitesse de montée du flux correspondant permet, pour des géométries identiques, de comparer les courants induits, et d'en chiffrer l'importance relative.
Pour réaliser ces mesures, il faut donc disposer de deux dynamiques différentes : l'une lente pour caractériser de façon quasi-statique le matériau, l'autre rapide pour développer des courants induits intenses [5].

Les signaux recueillis sur l'enroulement d'exploration lors de ces deux essais ont des amplitudes très différentes. Il est nécessaire dans ce cas de disposer d'un appareil ayant une gamme étendue de sensibilités.

Plusieurs méthodes industrielles ont été développées pour obtenir les courbes induction moyenne $\phi / S$ en fonction du champ d'excitation $N . I / L$. Ces méthodes peuvent être caractérisées à la fois par la nature de la variation du courant $I$ et par le mode d'obtention de l'intégrale de la force électromotrice recueillie pendant cette variation. Nous allons décrire les particularités de quelques-unes de ces techniques.

\subsection{Alimentation PARTICULIÈRE.}

2.3.1 Régime harmonique. - On impose une tension sinusoïdale aux bornes de la bobine. Sous certaines conditions, la f.é.m. issue des spires de mesure est sinusoïdale. La détermination de l'amplitude maximum du flux peut donc être faite simplement à partir de celle de la f.e.m.

Plusieurs précautions doivent être prises afin d'assurer une mesure représentative :

- limitation des courants induits. Les variations de flux doivent être lentes, ce qui implique une fréquence d'alimentation d'autant plus faible que le matériau est conducteur ;

- mesure du courant crête, le courant n'étant plus sinusoïdal dès l'apparition de la saturation ;

- limitation des chutes de tension internes dans le circuit, qu'elles soient de nature ohmique ou selfique, pour que le flux reste sinusoïdal. Cette condition est plus difficile à obtenir pour les fortes valeurs de l'induction. Dans ce cas seul l'usage d'un intégrateur permet une mesure du flux avec une précision acceptable.

2.3.2 Alimentation en échelon de tension. - Ce type d'alimentation est utilisé conjointement avec un galvanomètre balistique [2].

\subsection{AlimENTATIONS QUELCONQUES.}

2.4.1 Utilisation d'un fluxmètre. - La déviation d'un galvanomètre sans couple de rappel est proportionnelle à l'intégrale de la tension appliquée, avec une précision d'autant meilleure que la résistance totale du circuit est faible [4]. Les formes d'ondes peuvent être quelconques. Toutefois la faible impédance du circuit de mesure interdit les variations rapides de flux (courants induits supplémentaires). 
2.4.2 Utilisation d'un intégrateur électronique [6] [7]. Le signal $V_{\mathrm{s}}$ recueilli en sortie est de la forme :

$$
V_{\mathrm{s}}=-1 / T \int e \mathrm{~d} t
$$

pour une entrée $e(t)$ quelconque.

La constante de temps $T$ est fixée par les caractéristiques de composants passifs résistance et capacité, ce qui assure une bonne stabilité à long terme. De plus la caractéristique passe-bas de la fonction assure un filtrage des bruits de mesure de haute fréquence à valeur moyenne nulle.

2.4.3 Intégration numérique. - L'acquisition de la f.e.m. peut être faite directement et l'intégration réalisée hors ligne sur les valeurs acquises.

Cette méthode, exempte de dérive, présente cependant le défaut de nécessiter l'acquisition de tensions inférieures au millivolt, donc de prendre des précautions particulières de mesure et d'utiliser une préamplification. Par conséquent, une compensation de la tension de décalage de l'amplificateur doit être réalisée avec soin.

2.5 COMPARAISON DE MÉTHODES INDUSTRIELlES. Le tableau présenté sur la figure 3 résume les principales caractéristiques de plusieurs méthodes, la dernière étant celle que nous avons retenue.

\section{Description de l'appareillage.}

La figure 4 présente le schéma synoptique de l'installation.

L'appareillage de mesure couplé à un micro-calculateur se compose principalement :

- d'une source de courant contrôlée par le calculateur ;

- de cartes d'instrumentation programmables (amplificateurs ou intégrateurs) ;

- d'une carte d'interface avec le calculateur.

L'appareil peut contenir cinq cartes d'instrumentation, indifféremment amplificateurs ou intégrateurs, la programmation étant réalisée à partir du clavier, en précisant la nature de la carte et, suivant le cas la valeur du gain ou de la constante de temps choisie.

Deux catégories de cartes ont été développées :

- une comportant 4 intégrateurs à faible fuite dont les constantes de temps sont programmables dans des rapports 1,3 ou 10. Le choix de la capacité d'intégration permet l'adaptation de $T$ au domaine d'utilisation. Pour les mesures présentées une capacité de $0,1 \mu \mathrm{F}$ conduit à des valeurs de $2 \times 10^{-4} \mathrm{~s}$, $6 \times 10^{-4} \mathrm{~s}$ et $2 \times 10^{-3} \mathrm{~s}$;

- une comportant 3 amplificateurs d'instrumentation de gains programmés 3, 10, 30 ou 100 .

Le système d'acquisition possède 16 voies analogiques de 12 bits de résolution. Il peut fonctionner en accès direct de la mémoire du calculateur (D.M.A.)

\begin{tabular}{|c|c|c|c|c|c|}
\hline Alimentation & Appareil & Mesure & Stockage & Limites & Avantages \\
\hline $\begin{array}{l}\text { Tension } \\
\text { sinusoïdale }\end{array}$ & $\begin{array}{l}\text { Voltmètre } \\
\text { alternatif }\end{array}$ & $\begin{array}{l}\text { Un point de } \\
\text { la courbe de } \\
\text { de première } \\
\text { aimantation }\end{array}$ & Difficile & $\begin{array}{l}\text { * T.B.F. } \\
\text { * Faible } \\
\text { induction } \\
\text { *nan cycle }\end{array}$ & $\begin{array}{l}\text { Simplicité } \\
\text { de l'appareil } \\
\text { de mesure }\end{array}$ \\
\hline $\begin{array}{l}\text { Tension en } \\
\text { échelon }\end{array}$ & $\begin{array}{l}\text { Galvanomètre } \\
\text { ballistique }\end{array}$ & & $\begin{array}{l}\text { Mesure } \\
\text { "au vol" }\end{array}$ & $\begin{array}{l}\text { Courants } \\
\text { induits } \\
\text { intenses }\end{array}$ & \\
\hline $\begin{array}{l}\text { Alimentation } \\
\text { quelconque }\end{array}$ & $\begin{array}{l}\text { Fluxmètre } \\
\text { Grassot }\end{array}$ & $\begin{array}{l}\text { Lecture } \\
\text { continue } \\
\text { du flux }\end{array}$ & $\begin{array}{l}\text { une seule } \\
\text { mesure } \\
\text { stable }\end{array}$ & $\begin{array}{l}\text { * B.F(basse } \\
\text { impédance) } \\
\text { * Etalonnage } \\
\text { * Sensibilité }\end{array}$ & $\begin{array}{l}\text { Independant } \\
\text { du caractère } \\
\text { de la source }\end{array}$ \\
\hline$"$ & $\begin{array}{l}\text { Intégrateur } \\
\text { analogique }\end{array}$ & $"$ & $\begin{array}{l}\text { enregistre- } \\
\text {-ment } \\
\text { possible }\end{array}$ & Dérives & $\begin{array}{l}* \text { Sensibilité } \\
\text { modif iable } \\
\text { *Etalonnage }\end{array}$ \\
\hline$"$ & $\begin{array}{l}\text { Acquisition } \\
\text { intégration } \\
\text { numeriques }\end{array}$ & $\begin{array}{l}\text { Cycle } \\
\text { echantillonne }\end{array}$ & Automatique & $\begin{array}{l}\text { *Faibles } \\
\text { tensions } \\
\text { * Offset }\end{array}$ & $\begin{array}{l}\text { *pas dérive } \\
\text { *calcul } \\
\text { équations }\end{array}$ \\
\hline $\begin{array}{l}\text { Source de } \\
\text { courant } \\
\text { prog'rammable }\end{array}$ & $\begin{array}{l}\text { Integration } \\
\text { analogique } \\
\text { +acquisition }\end{array}$ & $"$ & " & & $\begin{array}{l}\text { * Sensibilite } \\
\text { program. } \\
\text { * Dérives } \\
\text { * Maitrise du } \\
\text { di/dt }\end{array}$ \\
\hline
\end{tabular}

Fig. 3. - Comparaison de plusieurs méthodes de mesure.

[Comparison between different measurement methods.] 


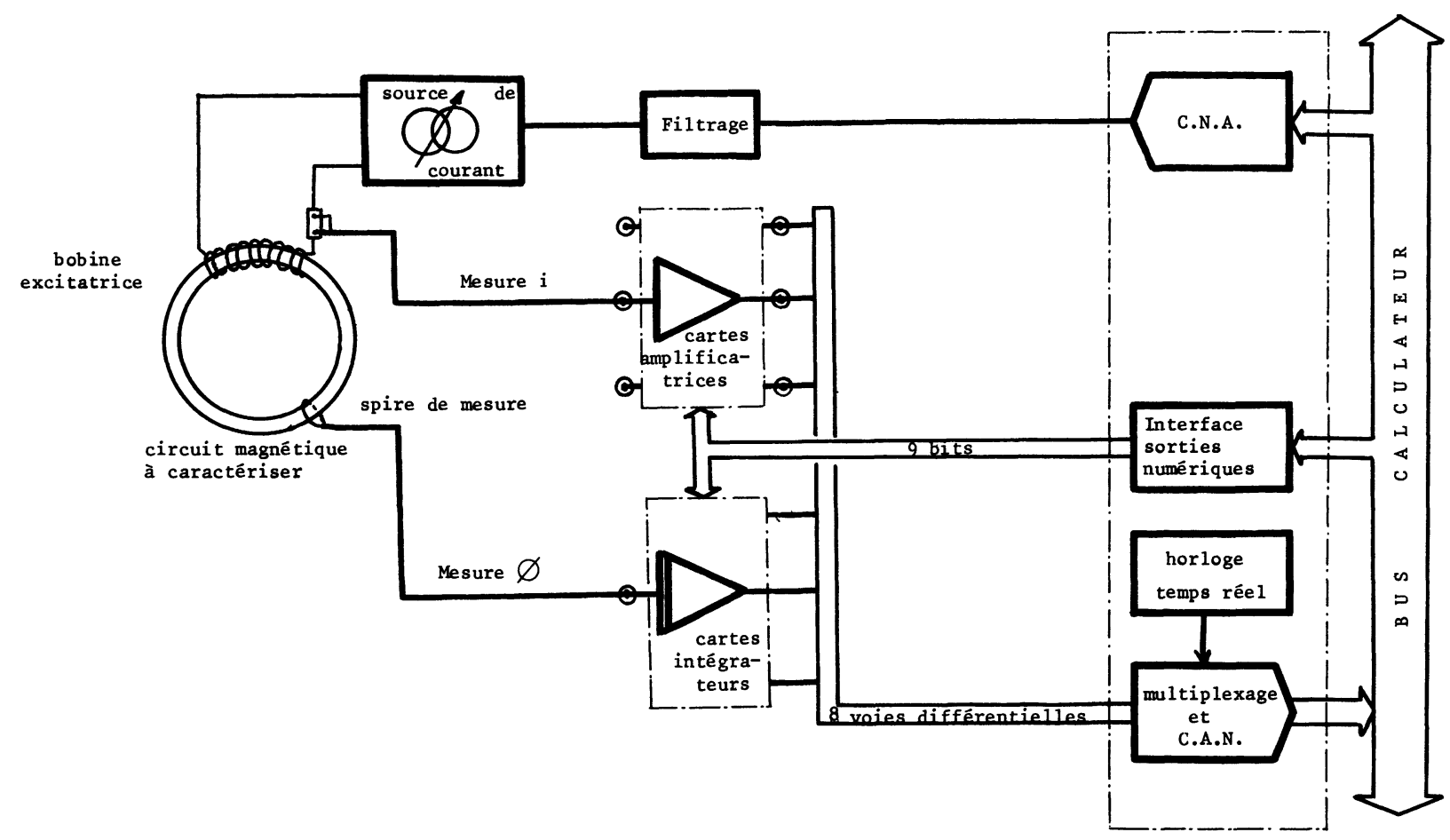

Fig. 4. - Schéma synoptique de l'installation.

[Block diagram of the measurement device.]

pour les mesures rapides (dans ce cas, l'ensemble de la chaîne autorise des périodes supérieures à $9 \mu \mathrm{s}$ entre deux mesures successives) ou en coup par coup sur demande du calculateur et autorisation d'une horloge temps réel.

La consigne de la source de courant peut être pilotée par le calculateur à travers un convertisseur numérique-analogique de résolution 12 bits suivi d'un filtrage passe-bas évitant toute variation brusque du courant. Cette source peut délivrer un courant maximum de $10 \mathrm{~A}$ en valeur positive ou négative, sous une tension maximum de $50 \mathrm{~V}$.

3.1 Mise EN cEUVRE D'UNE MESURE. - L'exemple présenté correspond à l'acquisition d'un cycle d'hystérésis complet relevé sur le circuit magnétique déjà décrit figure 2.

Il convient d'effectuer de façon quasi-simultanée la mesure échantillonnée :

- du courant d'excitation ;

- de l'intégrale de la f.e.m. développée aux bornes de la bobine de mesure.

Après la désaimantation du circuit par cycles décroissants, et la programmation des cartes, l'acquisition du courant $i$ et du flux $\phi$ se fait en deux temps (Fig. 5) :

- variation du courant d'excitation ;

- courant d'excitation nul.

Durant la première phase, la tension de sortie de l'intégrateur correspond à la somme algébrique
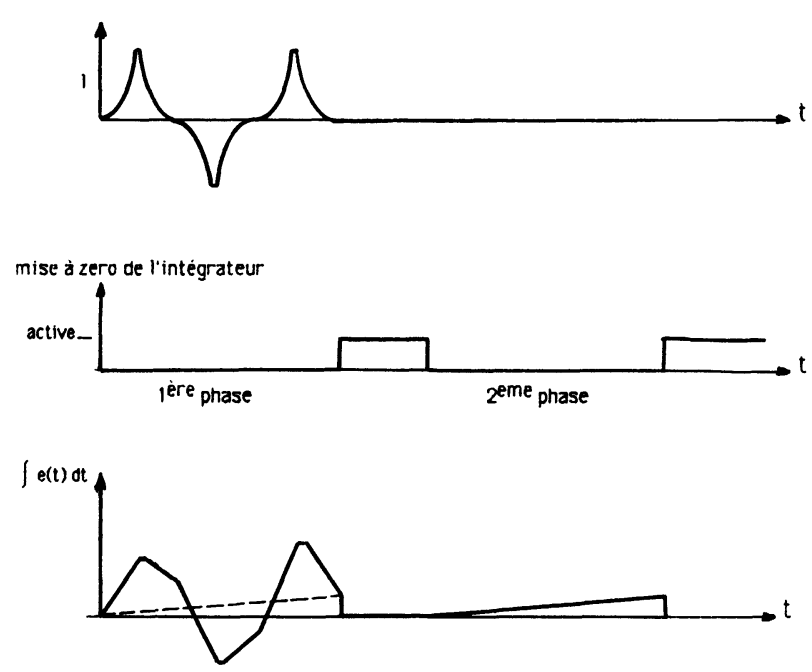

Fig. 5. - Evolution temporelle du courant, de la commande et de la sortie de l'intégrateur.

[Time-dependence of the current, voltage control and output voltage of the integrator.]

d'une grandeur proportionnelle au flux et d'une dérive de l'intégrateur.

Dans la deuxième phase, le circuit d'entrée n'ayant pas changé, la tension de sortie correspond à la mesure de la dérive seule. La valeur estimée du flux est obtenue en retranchant la dérive (signée) de la mesure obtenue dans la première phase. 


\subsection{PrÉCAutions DE MESURE.}

3.2.1 Compensation de la dérive. - Les condensateurs des montages intégrateurs sont à très faible fuite (polycarbonate). De plus les caractéristiques du circuit d'entrée restent inchangées pour toutes les mesures. Dans ces conditions, si on tient compte du courant de polarisation différentiel $i_{\mathrm{i} 0}$ et de la tension de décalage de l'étage d'entrée $v_{\mathrm{i} 0}$ de l'amplificateur opérationnel intégrateur la tension de sortie est donnée par la relation [8] :

$$
\begin{aligned}
v_{\mathrm{s}}=-1 / \mathrm{RC} \cdot \int e \cdot \mathrm{d} t-1 / \mathrm{RC} & \cdot \int v_{\mathrm{i} 0} \cdot \mathrm{d} t+ \\
& +1 / C \int i_{\mathrm{i} 0} \cdot \mathrm{d} t-v_{\mathrm{i} 0} .
\end{aligned}
$$

Seul le premier terme est utile ; les autres parasitent la mesure. Il faut donc soit en diminuer l'influence, soit les compenser.

Dans nos mesures les constantes de temps utilisées varient de $2 \times 10^{-4} \mathrm{~s}$ à $2 \times 10^{-3} \mathrm{~s}$, ce qui rend l'influence du dernier terme négligeable, même pour des tensions $e$ et $v_{\mathrm{i} 0}$ du même ordre de grandeur.

L'utilisation d'amplificateurs «bi-fet » rend négligeable l'influence du troisième terme.

L'effet du deuxième terme sera compensé en remarquant que la tension de décalage est fonction de la température, donc varie avec une constante de temps relativement élevée par rapport au temps de mesure. Tant que l'intégrateur n'est pas saturé, il y a addition de ce terme que nous avons appelé dérive et de la mesure proprement dite. Le mode de compensation est donc ainsi justifié.

Dans le cas où le signal $e$ fourni par la bobine est de trop faible amplitude, une amplification peut être nécessaire. La compensation dans ce cas englobe les tensions de décalage de l'amplificateur et de l'intégrateur, ce qui permet d'augmenter la résolution de la mesure de flux.
3.2.2 Vitesse de croissance du flux. - La vitesse de croissance du flux conditionne à la fois les courants induits et la sensibilité. Un bon compromis consiste à la maintenir approximativement constante, ce qui entraîne pour $i$ une variation croissante en fonction du temps.

3.2.3 Simultanéité des acquisitions de $\phi$ et $i$. - La non-simultanéité dans l'acquisition des échantillons de $\phi$ et $i$ ne doit pas engendrer d'erreur significative. Compte tenu de la vitesse d'évolution de ces deux grandeurs, des périodes d'échantillonnage choisies, les erreurs sur l'amplitude restent très inférieures au seuil de quantification du convertisseur de mesure.

\section{Le logiciel.}

Le logiciel offre à l'utilisateur le choix d'options prises dans des menus successifs. La figure 6 présente sous forme d'arbre les options principales.

\section{Mesures.}

Pour présenter des mesures réalisées avec l'appareil, deux matériaux de perméabilité et de conductivité différentes ont été testés :

- l'empilage de tôles au silicium décrit figure 2 ; - un tore de ferrite pouvant être considéré comme un très bon isolant électrique.

5.1 INFLUENCE DES COURANTS INDUITS. - La figure 7 présente la superposition de courbes de première aimantation enregistrées à des vitesses de montée du champ $H$ différentes (courbes $\mathrm{A}$ à $\mathrm{D}$ classées par ordre décroissant). On peut remarquer dans la zone linéaire une dispersion importante, ceci même pour des variations plus lentes que celles atteintes en flux imposé à $50 \mathrm{~Hz}$.

Cet essai permet de choisir la vitesse maximale de montée du flux lors du relevé de la caractéristique quasi-statique.

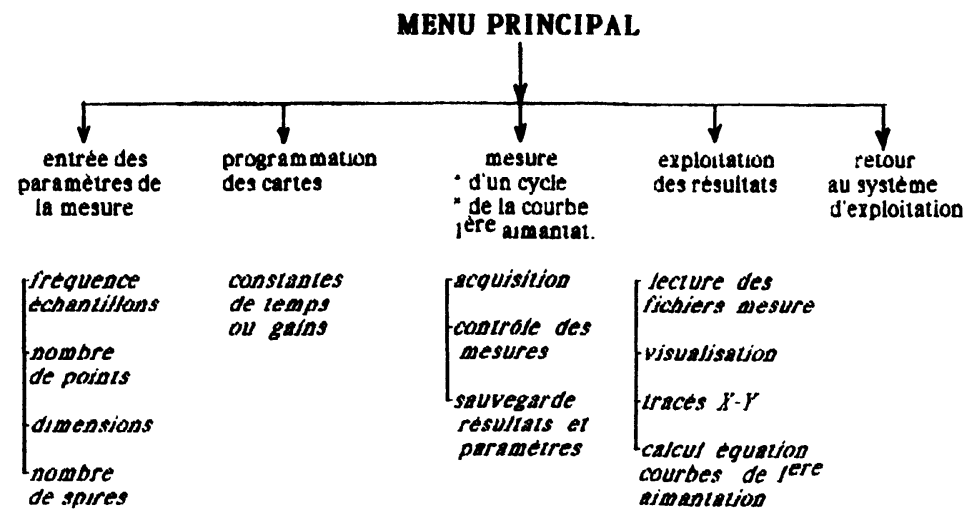

Fig. 6. - Choix principaux offerts par le logiciel à l'utilisateur.

[Main menu proposed to user's software.] 


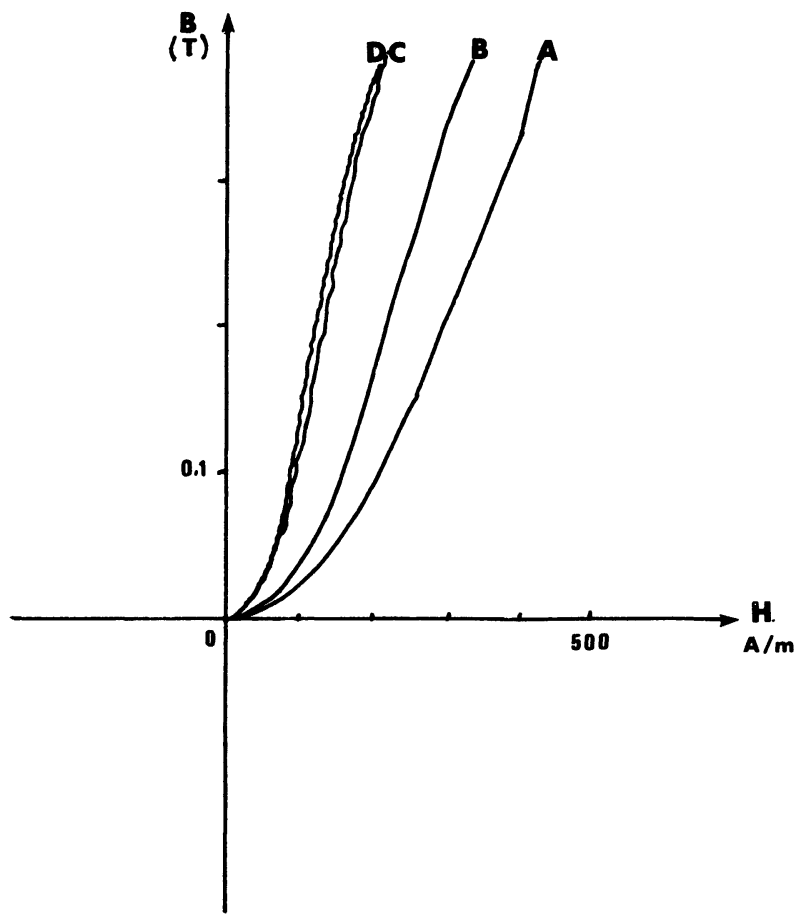

Fig. 7. - Courbes de première aimantation (tôles au silicium). Vitesse de montée du courant : A : $35 \mathrm{~A} / \mathrm{s} ; \mathrm{B}$ : $7 \mathrm{~A} / \mathrm{s} ; \mathrm{C}: 2 \mathrm{~A} / \mathrm{s} ; \mathrm{D}: 0,3 \mathrm{~A} / \mathrm{s}$.

[First magnetization curve (laminated circuit). Rate of current rise : $\mathrm{A}: 35 \mathrm{~A} / \mathrm{s} ; \mathrm{B}: 7 \mathrm{~A} / \mathrm{s} ; \mathrm{C}: 2 \mathrm{~A} / \mathrm{s} ; \mathrm{D}$ : $0,3 \mathrm{~A} / \mathrm{s}$.]

A titre de vérification, deux cycles ont été superposés pour le matériau isolant (Fig. 8). L'identité des résultats indique bien que le phénomène est lié à la circulation de courants induits.

Pour compléter cette vérification un enroulement supplémentaire fermé sur différentes résistances permet de se rendre compte, pour une vitesse constante de variation du courant, de l'utilisation de l'appareil en comparaison de courants induits à l'échelle de l'échantillon (Fig. 9).

5.2 CyCles QuASi-STATiQues. - En se plaçant dans les conditions déterminées précédemment pour pouvoir négliger l'influence des courants induits, plusieurs cycles d'amplitudes différentes ont été superposés, le matériau étant désaimanté entre chaque mesure.

On peut remarquer que tant pour le circuit feuilleté (cycles $A$ à $D$ de la Fig. 10) que pour le circuit ferrite (cycles $\mathrm{A}$ à $\mathrm{C}$ de la Fig. 11) les points extrêmes se situent sur la courbe de première aimantation.

\section{Conclusion.}

L'appareil décrit permet la mesure de flux et la caractérisation de matériaux magnétiques. Par l'usage de circuits programmables et d'un logiciel de

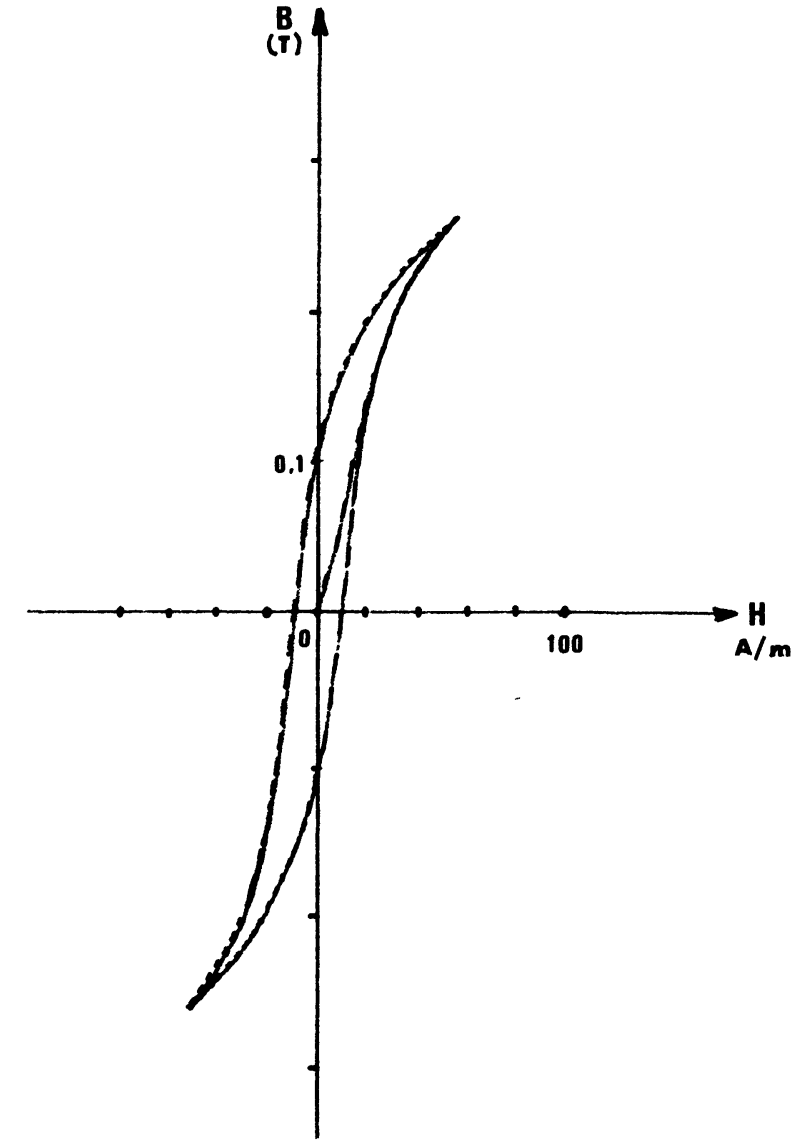

Fig. 8. - Superposition de 2 cycles à vitesse de montée du courant différente (rapport $1: 5$, tore de ferrite).

[Two hysteresis cycles corresponding to different rates of current rise (ratio $1: 5$, ferrite toroid).]

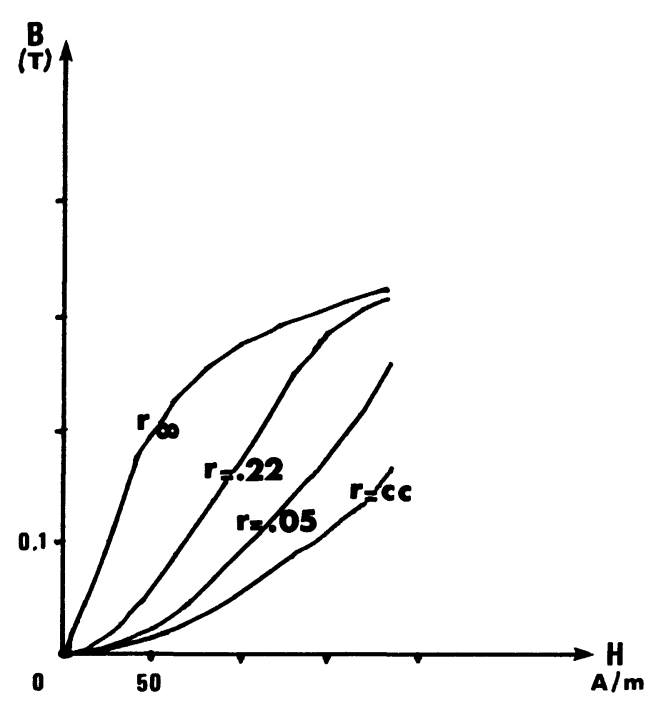

Fig. 9. - Courbes de première aimantation avec circuit auxiliaire fermé sur une résistance $r$ (tore de ferrite).

[First magnetization curve with an auxiliary winding shorted by a resistor (ferrite toroid).] 


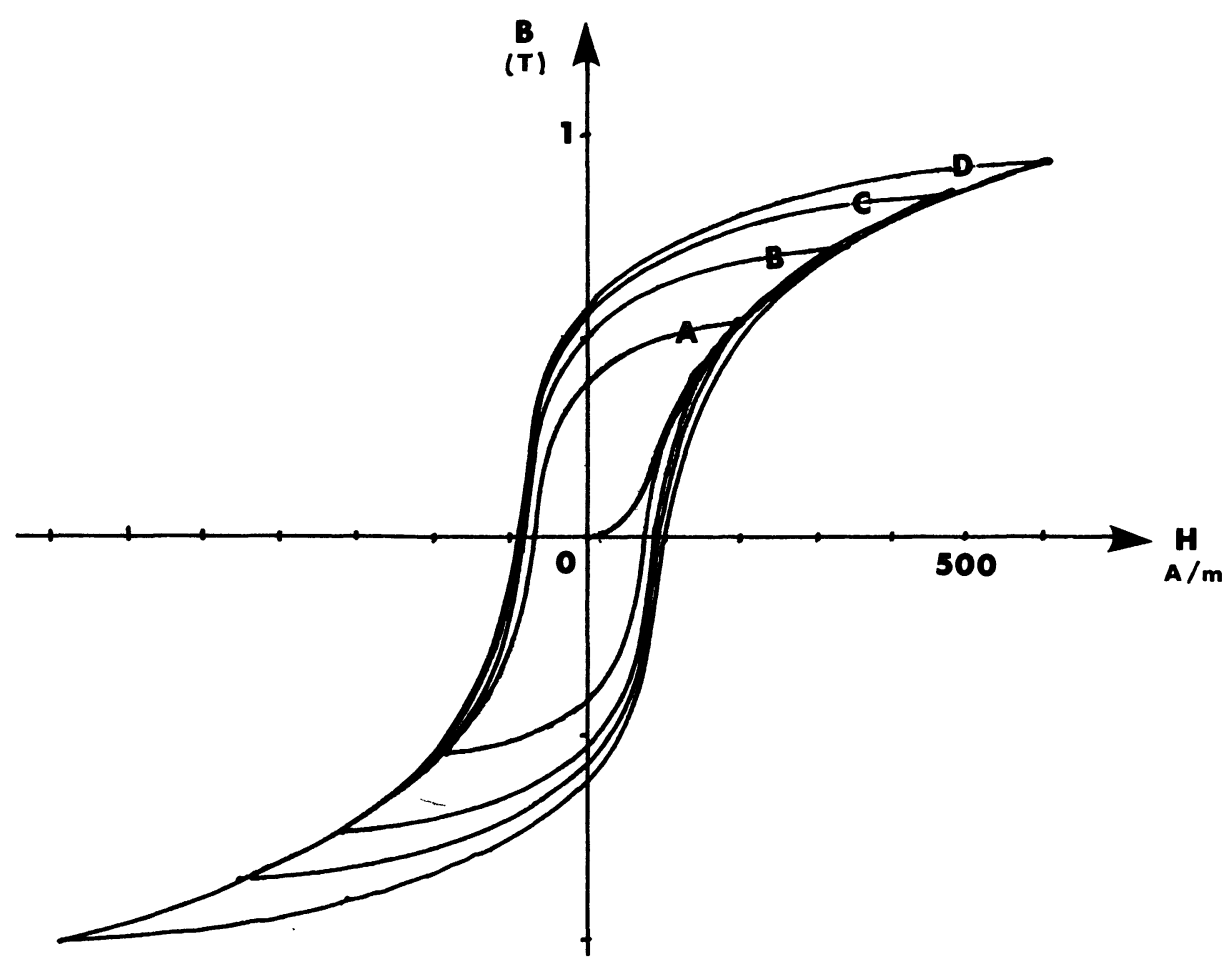

Fig. 10. - Superposition de cycles quasi-statiques d'amplitude maximum croissante (A à $\mathrm{D}$, tôles au silicium). [Four quasi-static hysteresis cycles with increasing amplitude (from A to D, laminated circuit).]

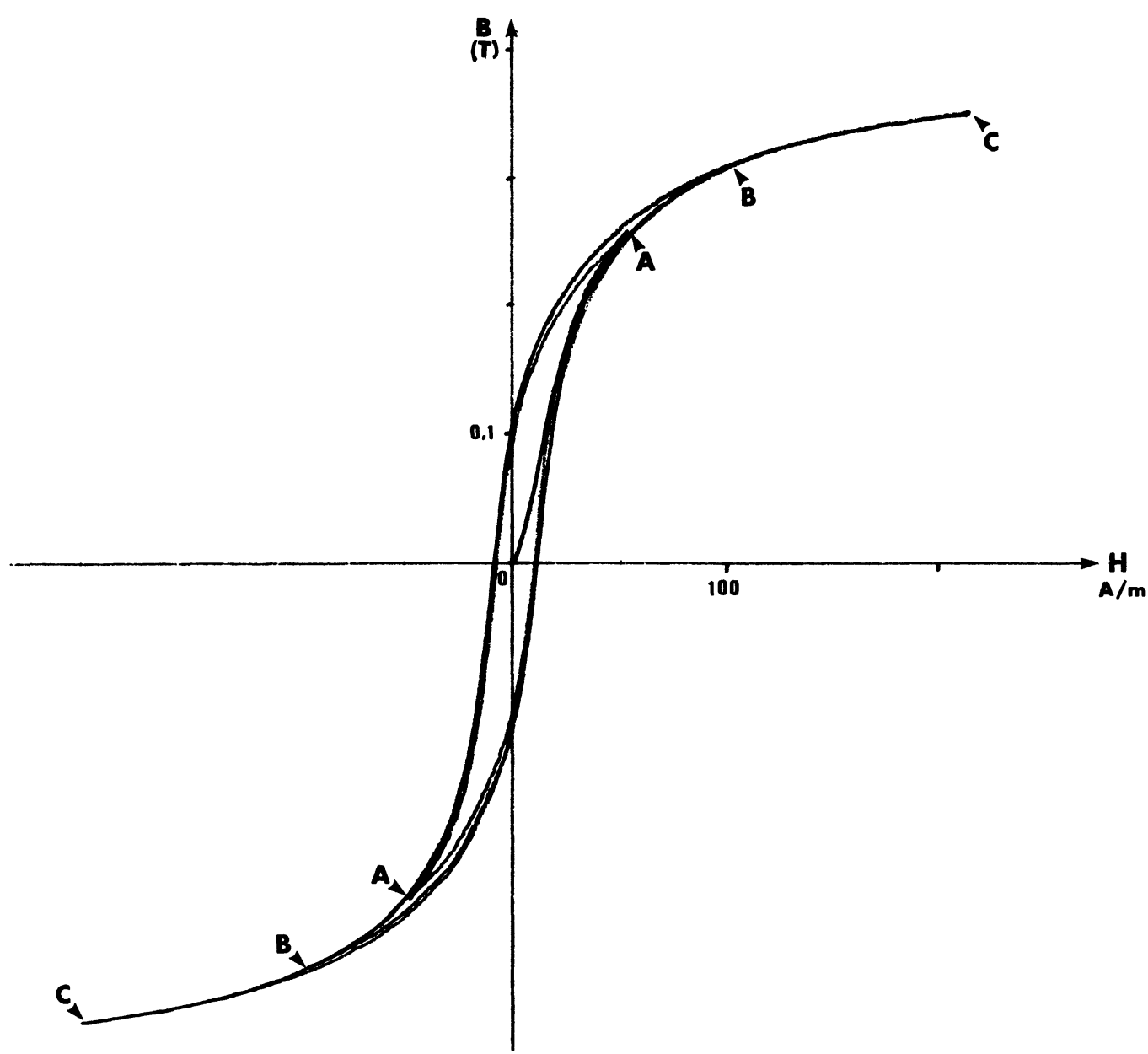

Fig. 11. - Superposition de cycles quasi-statiques d'amplitude maximum croissante (A à C, tore de ferrite). [Three quasi-static hysteresis cycles with increasing amplitude (from A to $\mathrm{C}$, ferrite toroid).] 
commande associé, par la compensation de dérive des circuits de mesure, des problèmes de taille et de nature différentes ont pu être résolus.

Pour des fabricants ou des utilisateurs de matériaux magnétiques, l'appareil permet l'acquisition, le tracé de cycles d'hystérésis quasi-statiques et l'évaluation de leurs conditions de validité.

Il offre également la possibilité de contrôler des circuits de géométries identiques présentant des caractéristiques de conduction différentes (conductibilité, qualité de l'isolation de circuits feuilletés, soudures...).
Une mesure des pertes en régime sinusoïdal $50 \mathrm{~Hz}$ étant insuffisante pour caractériser les matériaux de construction des machines alimentées par des convertisseurs, des auteurs [9] ont montré que les pertes des matériaux magnétiques pouvaient être caractérisées lors de variations d'induction trapézoïdales.

D'ores et déjà, notre appareil peut réaliser des mesures dans ces conditions, et s'adapter, sans remettre en cause sa construction, à des formes d'ondes quelconques.

\section{Bibliographie}

[1] SABonnadière, J. C., Conception des machines électriques: la modélisation facilite l'optimisation. Rev. Gen. Electr. n 3, mars 1987 (article de synthèse).

[2] ZiJlstra, H., Experimental methods in magnetism (North-Holland Publishing Company, Amsterdam) 1967.

[3] SANFORD, R. and COOTER, I., Basic magnetic quantities and the measurement of the magnetic properties of materials, National Bureau of Standards Monograph 47, U.S. Department of commerce (1962).

[4] Elnekave, N., Mesures magnétiques, Techniques de l'ingénieur.

[5] Kedous, A., Lebouc, D. et Brissonneau, P., Etude des pertes dans des tôles magnétiques soumises à des variations d'induction $\mathrm{B}(\mathrm{t})$ de forme trapézoïdale, Revue Phys. Appl. 21 (1986).

[6] WozeK, J., Etude et réalisation d'un appareil d'enregistrement du cycle d'hystérésis des ferro et ferrimagnétiques utilisant la technique de l'échantillonnage, Thèse d'université, Paris-Sud 1976.

[7] Henryon, M., Viard, M., Durupt, S., DunstetTER, R., Appareil automatique de contrôle non destructif des fontes G.S., Fonderie, Fondeurs d'aujourd'hui 51 (1986).

[8] STouT, D., Handbook of operational amplifier circuit design (Kaufman Ed.) 1976.

[9] Allano, S., Lebouc, A. et Perret, R., Pertes dans les circuits magnétiques, Rev. Gen. Electr. $n^{\circ} 3$ (mars 1987). 